\title{
Relación entre lectura de los escritos de Elena G. de White y el compromiso cristiano en feligreses adventistas
}

\section{Relation between reading of the writings of Ellen White and christian commitment in seventh-day adventist members}

\author{
Stephenson Celant ${ }^{1 \mathrm{a}}$ y Marcelo Zanga Céspedes ${ }^{2 \mathrm{a}}$ \\ Andrews University, Michigan, Estados Unidos de Norteamerica ${ }^{1}$ \\ Universidad Peruana Unión, Lima, Perú ${ }^{2}$ \\ (iD) https://orcid.org/0000-0002-0616-6955 \\ (iD https://orcid.org/0000-0003-1244-30552
}

Recibido: 15 de diciembre de 2019

Aceptado: 11 de mayo de 2020

\section{Resumen}

Este estudio tiene por objetivo determinar la relación entre la lectura de los escritos de Elena G. de White y el compromiso cristiano en feligreses adventistas de Ecuador.

Participaron 120 personas pertenecientes a la iglesia adventista y contestaron dos instrumentos, el primero relacionado al nivel de lectura y compresión y el segundo relacionado al compromiso cristiano, el cual tomó en cuenta las dimensiones de "comunión," "relacionamiento" y "misión." Los resultados de esta investigación muestran un valor de significancia $\mathrm{p}=, 030<\alpha=, 05$, lo que indica que la relación entre las variables es significativa; y el coeficiente de correlación de Spearman $=, 557$, lo que señala una correlación media entre las variables; por lo tanto se concluye que la lectura de los libros de Elena G. de White se relaciona significativamente con el nivel de compromiso cristiano.

Palabras claves: Lectura, compromiso, Elena G. de White, iglesia adventista

\begin{abstract}
This study aims to determine the relationship between the reading of the writings of Ellen White and christian commitment in Adventist people in Ecuador. 120 seventh-day adventist church members participated and answered two instruments: the first related to the level of reading and understanding and the second related to christian commitment, which took into account the dimensions of "communion," "relationship" and "mission." The results of this investigation show a value of significance $p=.030<\alpha=.05$, which indicates that the relationship between the variables is significant; and the Spearman
\end{abstract}

${ }^{\mathrm{a} C o r r e s p o n d e n c i a ~ a ~ l o s ~ a u t o r e s: ~}$

E-mail: celant@andrews.edu y marcelozanga @ upeu.edu.pe 
correlation coefficient $=$, 557, which indicates an average correlation between the variables; therefore it is concluded that the reading of Ellen White books is significantly related to the level of christian commitment.

Keywords: reading, commitment, Ellen White, Seventh-day Adventist Church

\section{Introducción}

Los cristianos aceptan que la Biblia es la única regla de fe y práctica, que tiene el propósito de hacer al creyente sabio para la salvación (2 Tim 3:15) y su mensaje señala a una recompensa inmediata: la bienaventuranza, pues Juan declara "Bienaventurado el que lee, y los que oyen las palabras de esta profecía, y guardan las cosas en ella escritas; porque el tiempo está cerca" (Ap 3:1). Por lo tanto, leer las Sagradas Escrituras es una actividad necesaria.

En palabras de Presser (2011) la lectura regular de las Sagradas Escrituras permite "que nuestra percepción de los hechos que vivimos a diario sea madura y a la vez fortalece nuestro juicio, porque nuestros pensamientos y sentimientos están orientados por la promesa de la Biblia”. Elena G. de White lo expreso de manera más profunda señalando que: "Si se estudiara la Palabra de Dios como se debe, los hombres tendrían una grandeza de espíritu, una nobleza de carácter y una firmeza de propósito que raramente pueden verse en estos tiempos" (2018). Es decir, la lectura y el estudio de la Biblia abre la mente de los que la leen, y, más aun, produce la formación y transformación del carácter.

La lectura de la Biblia era central en la vida y ministerio de Jesús. Él señaló que sus genuinos seguidores son los que viven de toda palabra que sale de la boca de Dios (Mt 4:4), que escuchan sus palabras y las ponen en práctica (Timm, 2009). Cristo mismo era un fiel lector de las Sagradas Escrituras. Dice el relato bíblico que él 'Llegó a Nazaret, donde se había criado, y según su costumbre, entró en la sinagoga el día de reposo, y se levantó a leer (Lc 4:16). Siguiendo el ejemplo de Jesús, Asmat (2012) asevera que "la lectura de la Biblia siguió ocupando una parte central del culto cristiano." Entonces, la lectura de la Biblia que transforma a los seres humanos, debería ser una actividad de práctica diaria.

No obstante, los datos actuales muestran que la cantidad de lectores de la Biblia está disminuyendo. Según Christianity Today, el número de personas que afirmaron haber leído la Biblia para sentirse más cerca de Dios se redujo del "64 por ciento en 2011, cayó al 55 por ciento en 2012, luego volvió a establecerse en 55 por ciento en 2016" (2018). En cuanto a los jóvenes, la misma investigación menciona que "los millenials cristianos 
creen menos que cualquier otra generación, que la Biblia contiene todo lo que una persona necesita para vivir una vida significativa o plena. Más de la mitad $(55 \%)$ son neutrales o escépticos de la Biblia."

La Biblia llegó hasta nosotros a través del proceso de revelación e inspiración, por medio del cual Dios capacita a un hombre o una mujer de su especial elección para recibir y comunicar en forma precisa, competente y fidedigna los mensajes de Dios para su pueblo (Coon, 2017). Tanto en el Antiguo como en el Nuevo testamento, Dios reveló sus mensajes a sus siervos los profetas (Heb.1:1). Aunque Dios usó muchos métodos o formas de comunicación con la raza humana, los profetas fueron "el canal más visible en el sistema de la comunicación de Dios" (Douglass 2003); porque el Señor mismo declara que no hará nada sin antes comunicarlo a sus siervos los profetas (Amos 3:7).

El don de profecía es uno de los dones que Dios otorga a su iglesia (1 Co 12:10). Y aunque muchos creen que el don profético cesó al final de la era apostólica, la Palabra de Dios indica que ese don sería necesario para la iglesia y proveería dirección divina durante las crisis finales para guiar la iglesia a fin de alcanzar "la unidad de la fe" (Ef 4: 13); razón por la cual el apóstol Pablo amonesta a los creyentes: "no apaguéis al Espíritu. No menospreciéis las profecías" (1 Tes 5: 19, 20). Ese don profético, se manifiesta sobre toda carne, hombres o mujeres sin distinción de género.

Por su parte, la Iglesia Adventista del Séptimo Día, luego del gran chasco del 22 de octubre de 1844, acepta y predica que Dios otorgó al movimiento adventista el don profético en la persona de Elena Harmon. Este don es una señal identificadora de la iglesia remanente y que es una permanente y autorizada fuente de verdad que proporciona consuelo, dirección, instrucción y corrección a la iglesia.

El ministerio de Elena de White influenció en diversas áreas, según Burchfield menciona que "Las visiones proféticas de Elena G. de White les mantuvieron en un cuerpo coherente que hoy es la Iglesia Adventista del Séptimo Día” (Burchfield, 1996), Knight menciona que "los escritos de Elena G. de White han sido una bendición para las incontables personas que los han leído durante los últimos ciento cincuenta años" (Knight, 2004). Al mismo tiempo, Douglass menciona que "el ministerio profético de Elena G. de White y el surgimiento de la Iglesia Adventista del Séptimo Día son inseparables. Tratar de entender el uno sin el otro haría que cada uno fuese inteligible e indescubrible" (Douglass, 2003). 
Según un estudio realizado a los miembros de la Iglesia Adventista del Séptimo día en 193 iglesias en Estados Unidos, donde participaron 8200 personas de los cuales 2.848 leen los libros de Espíritu de Profecía (5.375 no lo hacen), concluyeron que:

$85 \%$ de lectores sintieron que tienen una mejor relación íntima con Cristo, mientras que solo $59 \%$ de los no-lectores sintieron lo mismo. $82 \%$ tenían la seguridad de estar bien con Dios contra 59\%; 65\% usan sus dones espirituales contra 49\%; 72\% participan en evangelismo público contra 62\%; 73\% involucrado en la testificación contra $49 \% ; 82 \%$ da estudio biblico contra 47\%; $46 \%$ han ganado un alma en los últimos 3 años contra 33\% etc. (Des Cumings, 1982).

A pesar que este estudio revela que los que leen los escritos de Elena G. de White han experimentado un mayor compromiso con Dios, en investigaciones más recientes se puede apreciar una baja considerable en cuento a la lectura de sus escritos. Joseph Kidder investigó sobre la frecuencia de las prácticas devocionales entre los miembros en Sudamérica y concluyó que, la frecuencia de lectura de los escritos de Elena G. de White diariamente sólo llega al 14\%; los que leen una vez por semana llegan al $29 \%$ y los que nunca leen corresponde al 57\%. (Kidder, 2007)

Por su parte, Alberto Timm, comenta el resultado de la investigación que Bailey Gillespie realizó con jóvenes de las escuelas secundarias adventistas del séptimo día en América del Norte. Este estudio reveló que hay una disminución significativa de los que leían a Elena G. White una vez a la semana. La primera encuesta, en 1990, indicó sólo el $13 \%$ de los estudiantes lo hacían, la segunda, encuesta realizada el 2000, mostró que sólo era $6 \%$ lo hizo y la tercera, en el 2010, mostró únicamente un 2\%” (Timm, 2004).

Tomando en cuenta los datos anteriores se puede decir que el compromiso cristiano de los profesos seguidores de Cristo está disminuyendo. A nivel mundial, la Biblia deja de ser relevante para muchos y entre los adventistas los escritos de Elena G. de White dejan de ser leídos. Por ello, este estudio tuvo como objetivo determinar la relación que existe relación entre la lectura de los escritos de Elena G. de White y compromiso cristiano de los feligreses adventistas de diferentes iglesias en Chone, Ecuador.

\section{Método}

La investigación fue de tipo cuantitativo porque las variables son observables, es decir, medibles. Es descriptiva porque describe los hechos tal como son observados. Es correlacional porque estudia las relaciones entre variables dependientes e independientes. 
Tuvo un diseño no-experimental ya que el investigador no manipuló ninguna de las variables (Richard, 2013).

En relación a la población, de los 120 miembros que constituían la población, solo participaron 108 miembros de las iglesias adventistas del séptimo día en Chone, Ecuador. La muestra fue no probabilística, porque se invitó a todos los miembros de las iglesias, pero solo se pudo tomar en cuenta a los que voluntariamente decidieron participar.

El instrumento consistió en un cuestionario-de carácter anónimo-, el cual comprendió 47 ítems divididos en dos secciones. La sección uno estaba subdivida en dos partes, la primera parte era el test de comprensión lectora que incluía la información general de los participantes y la segunda parte del instrumento tomó en cuenta la variable compromiso cristiano.

Previo a la aplicación del instrumento, se realizó una prueba piloto en un distrito pastoral en la ciudad de Lima, con la participación de 34 personas, para verificar si el instrumento necesitaba algún tipo de ajustes. También se recurrió a un jurado experto para la evaluación de los instrumentos.

En la tabla 1 se presenta los datos relacionados a las características de género donde el $44.4 \%$ de los participantes fueron varones, mientras que el $53.7 \%$ fueron mujeres. Y en relación a los grupos de edad, descrito en la tabla 2, el mayor porcentaje se concentró entre 21 a 40 años llegando a sumar el $53.7 \%$ del total.

Tabla 1

Características de género

\begin{tabular}{llrrrr}
\hline & & Frecuencia & Porcentaje & $\begin{array}{c}\text { Porcentaje } \\
\text { válido }\end{array}$ & $\begin{array}{c}\text { Porcentaje } \\
\text { acumulado }\end{array}$ \\
\hline Válido & Masculino & 48 & 44.4 & 45.3 & 45.3 \\
& Femenino & 58 & 53.7 & 54.7 & 100.0 \\
& Total & 106 & 98.1 & 100.0 & \\
Perdidos & Sistema & 2 & 1.9 & & \\
Total & & 108 & 100.0 & & \\
\hline
\end{tabular}

Fuente: Elaboración propia 


\section{Tabla 2}

Edad

\begin{tabular}{llrrrr}
\hline & & Frecuencia & Porcentaje & $\begin{array}{c}\text { Porcentaje } \\
\text { válido }\end{array}$ & $\begin{array}{c}\text { Porcentaje } \\
\text { acumulado }\end{array}$ \\
\hline Válido & $14-20$ & 13 & 12.0 & 14.1 & 14.1 \\
& $21-30$ & 36 & 33.3 & 39.1 & 53.3 \\
& $31-40$ & 22 & 20.4 & 23.9 & 77.2 \\
& $41-50$ & 14 & 13.0 & 15.2 & 92.4 \\
& $51-60$ & 6 & 5.6 & 6.5 & 98.9 \\
& 60 o más & 1 & .9 & 1.1 & 100.0 \\
Perdidos & Total & 92 & 85.2 & 100.0 & \\
Total & Sistema & 16 & 14.8 & & \\
\hline Fuente: & & 108 & 100.0 & & \\
\hline
\end{tabular}

Fuente: Elaboración propia

\section{Resultados}

Los datos recolectados se procesaron con el programa SPSS y se hallaron los siguientes resultados:

\section{Tabla 3}

Relación entre lectura de los libros de Elena de White y compromiso cristiano

\begin{tabular}{|c|c|c|c|c|c|c|c|c|c|c|c|c|}
\hline \multirow{3}{*}{$\begin{array}{l}\text { Lectura de los libros del } \\
\text { espíritu de profecía }\end{array}$} & \multicolumn{12}{|c|}{ Compromiso cristiano } \\
\hline & \multicolumn{2}{|c|}{$\begin{array}{l}\text { Nivel muy } \\
\text { bajo }\end{array}$} & \multicolumn{2}{|c|}{ Nivel bajo } & \multicolumn{2}{|c|}{$\begin{array}{c}\text { Nivel } \\
\text { moderado }\end{array}$} & \multicolumn{2}{|c|}{ Nivel alto } & \multicolumn{2}{|c|}{$\begin{array}{l}\text { Nivel muy } \\
\text { alto }\end{array}$} & \multicolumn{2}{|c|}{ Total } \\
\hline & $\mathrm{n}$ & $\%$ & $\mathrm{n}$ & $\%$ & $\mathrm{n}$ & $\%$ & $\mathrm{n}$ & $\%$ & $\mathrm{~N}$ & $\%$ & $\bar{n}$ & $\%$ \\
\hline Nivel muy alto & 0 & $0,0 \%$ & 0 & $0,0 \%$ & 0 & $0,0 \%$ & 1 & $33,3 \%$ & 2 & $66,7 \%$ & 3 & $100,0 \%$ \\
\hline Nivel alto & 4 & $8,0 \%$ & 7 & $14,0 \%$ & 9 & $18,0 \%$ & 15 & $30,0 \%$ & 15 & $30,0 \%$ & 50 & $100,0 \%$ \\
\hline Nivel moderado & 1 & $2,6 \%$ & 13 & $33,3 \%$ & 14 & $35,9 \%$ & 8 & $20,5 \%$ & 3 & $7,7 \%$ & 39 & $100,0 \%$ \\
\hline Nivel bajo & 4 & $57,1 \%$ & 1 & $14,3 \%$ & 1 & $14,3 \%$ & 1 & $14,3 \%$ & 0 & $0,0 \%$ & 7 & $100,0 \%$ \\
\hline Nive muy bajo & 4 & $80,0 \%$ & 0 & $0,0 \%$ & 0 & $0,0 \%$ & 1 & $20,0 \%$ & 0 & $0,0 \%$ & 5 & $100,0 \%$ \\
\hline Total & 13 & $12,5 \%$ & 21 & $20,2 \%$ & 24 & $23,1 \%$ & 26 & $25,0 \%$ & 20 & $19,2 \%$ & 104 & $100,0 \%$ \\
\hline
\end{tabular}

Chi-cuadrado de Pearson $=28,189$ g.l. $=16 \quad p=, 030 *<.05$

Correlación de Spearman $=.557 * *$ y $p=, 000<.05$

*. El estadístico de chi-cuadrado es significativo en el nivel ,05.

**. La correlación es significativa en el nivel 0,01 (2 colas). 


\section{Tabla 4}

Relación entre la lectura rápida de los libros del espíritu de profecía con el compromiso cristiano

\begin{tabular}{|c|c|c|c|c|c|c|c|c|c|c|c|c|}
\hline \multirow[b]{3}{*}{ Lectura rápida } & \multicolumn{12}{|c|}{ Compromiso cristiano } \\
\hline & \multicolumn{2}{|c|}{$\begin{array}{l}\text { Nivel muy } \\
\text { bajo }\end{array}$} & \multicolumn{2}{|c|}{ Nivel bajo } & \multicolumn{2}{|c|}{$\begin{array}{c}\text { Nivel } \\
\text { moderado }\end{array}$} & \multicolumn{2}{|c|}{ Nivel alto } & \multicolumn{2}{|c|}{$\begin{array}{l}\text { Nivel muy } \\
\text { alto }\end{array}$} & \multicolumn{2}{|c|}{ Total } \\
\hline & $n$ & $\%$ & $\mathrm{n}$ & $\%$ & $\mathrm{n}$ & $\%$ & $\mathrm{~N}$ & $\%$ & $\mathrm{n}$ & $\%$ & $\mathrm{~N}$ & $\%$ \\
\hline Nivel muy alto & 3 & $42,9 \%$ & 2 & $28,6 \%$ & 0 & $0,0 \%$ & 1 & $14,3 \%$ & 1 & $14,3 \%$ & 7 & $100,0 \%$ \\
\hline Nivel alto & 5 & $22,7 \%$ & 12 & $54,5 \%$ & 2 & $9,1 \%$ & 2 & $9,1 \%$ & 1 & $4,5 \%$ & 22 & $100,0 \%$ \\
\hline Nivel moderado & 2 & $6,9 \%$ & 4 & $13,8 \%$ & 16 & $55,2 \%$ & 3 & $10,3 \%$ & 4 & $13,8 \%$ & 29 & $100,0 \%$ \\
\hline Nivel bajo & 1 & $2,9 \%$ & 2 & $5,9 \%$ & 8 & $23,5 \%$ & 20 & $58,8 \%$ & 3 & $8,8 \%$ & 34 & $100,0 \%$ \\
\hline Nive muy bajo & 2 & $12,5 \%$ & 1 & $6,3 \%$ & 0 & $0,0 \%$ & 1 & $6,3 \%$ & 12 & $75,0 \%$ & 16 & $100,0 \%$ \\
\hline Total & 13 & $12,0 \%$ & 21 & $19,4 \%$ & 26 & $24,1 \%$ & 27 & $25,0 \%$ & 21 & $19,4 \%$ & 108 & $100,0 \%$ \\
\hline
\end{tabular}

Chi-cuadrado de Pearson =102,499 g.l. = $16 \quad p=, 030 *<.05$

Correlación de Spearman $=.-, 597 * *$ y $p=, 000<.05$

*. El estadístico de chi-cuadrado es significativo en el nivel ,05.

**. La correlación es significativa en el nivel 0,01 (2 colas).

\section{Tabla 5}

Relación entre la lectura mecánica de los libros del espíritu de profecía con el compromiso cristiano

\begin{tabular}{|c|c|c|c|c|c|c|c|c|c|c|c|c|}
\hline \multirow[b]{3}{*}{ Lectura mecánica } & \multicolumn{12}{|c|}{ Compromiso cristiano } \\
\hline & \multicolumn{2}{|c|}{ Nive muy bajo } & \multicolumn{2}{|c|}{ Nivel bajo } & \multicolumn{2}{|c|}{$\begin{array}{c}\text { Nivel } \\
\text { moderado }\end{array}$} & \multicolumn{2}{|c|}{ Nivel alto } & \multicolumn{2}{|c|}{$\begin{array}{l}\text { Nivel muy } \\
\text { alto }\end{array}$} & \multicolumn{2}{|c|}{ Total } \\
\hline & $\mathrm{n}$ & $\%$ & $\mathrm{n}$ & $\%$ & $\mathrm{n}$ & $\%$ & $\mathrm{n}$ & $\%$ & $\mathrm{n}$ & $\%$ & $\mathrm{n}$ & $\%$ \\
\hline Nivel muy alto & 0 & $0,0 \%$ & 2 & $20,0 \%$ & 1 & $10,0 \%$ & 1 & $10,0 \%$ & 6 & $60,0 \%$ & 10 & $100,0 \%$ \\
\hline Nivel alto & 0 & $0,0 \%$ & 3 & $20,0 \%$ & 4 & $26,7 \%$ & 5 & $33,3 \%$ & 3 & $20,0 \%$ & 15 & $100,0 \%$ \\
\hline Nivel moderado & 4 & $7,1 \%$ & 12 & $21,4 \%$ & 13 & $23,2 \%$ & 16 & $28,6 \%$ & 11 & $19,6 \%$ & 56 & $100,0 \%$ \\
\hline Nivel bajo & 5 & $23,8 \%$ & 3 & $14,3 \%$ & 8 & $38,1 \%$ & 4 & $19,0 \%$ & 1 & $4,8 \%$ & 21 & $100,0 \%$ \\
\hline Nive muy bajo & 4 & $66,7 \%$ & 1 & $16,7 \%$ & 0 & $0,0 \%$ & 1 & $16,7 \%$ & 0 & $0,0 \%$ & 6 & $100,0 \%$ \\
\hline Total & 13 & $12,0 \%$ & 21 & $19,4 \%$ & 26 & $24,1 \%$ & 27 & $25,0 \%$ & 21 & $19,4 \%$ & 108 & $100,0 \%$ \\
\hline
\end{tabular}

Chi-cuadrado de Pearson $=39,911$ g.l. $=16 \quad p=, 001 *<05$

Correlación de Spearman $=, 575 * *$ y $p=, 000<.05$

*. El estadístico de chi-cuadrado es significativo en el nivel ,05.

**. La correlación es significativa en el nivel 0,01 (2 colas).

\section{Tabla 6}

Relación entre la frecuencia de lectura de los libros del espíritu de profecía con el compromiso cristiano

\begin{tabular}{|c|c|c|c|c|c|c|c|c|c|c|c|c|}
\hline \multirow[b]{3}{*}{ Frecuencia de lectura } & \multicolumn{12}{|c|}{ Compromiso cristiano } \\
\hline & \multicolumn{2}{|c|}{ Nive muy bajo } & \multicolumn{2}{|c|}{ Nivel bajo } & \multicolumn{2}{|c|}{$\begin{array}{c}\text { Nivel } \\
\text { moderado }\end{array}$} & \multicolumn{2}{|c|}{ Nivel alto } & \multicolumn{2}{|c|}{$\begin{array}{l}\text { Nivel muy } \\
\text { alto }\end{array}$} & \multicolumn{2}{|c|}{ Total } \\
\hline & $\mathrm{n}$ & $\%$ & $\mathrm{~N}$ & $\%$ & $\mathrm{n}$ & $\%$ & $\mathrm{n}$ & $\%$ & $\mathrm{n}$ & $\%$ & $\mathrm{n}$ & $\%$ \\
\hline Nivel muy alto & 0 & $0,0 \%$ & 2 & $11,8 \%$ & 4 & $23,5 \%$ & 4 & $23,5 \%$ & 7 & $41,2 \%$ & 17 & $100,0 \%$ \\
\hline Nivel alto & 3 & $8,3 \%$ & 5 & $13,9 \%$ & 9 & $25,0 \%$ & 11 & $30,6 \%$ & 8 & $22,2 \%$ & 36 & $100,0 \%$ \\
\hline Nivel moderado & 4 & $11,4 \%$ & 11 & $31,4 \%$ & 10 & $28,6 \%$ & 8 & $22,9 \%$ & 2 & $5,7 \%$ & 35 & $100,0 \%$ \\
\hline Nivel bajo & 4 & $36,4 \%$ & 3 & $27,3 \%$ & 2 & $18,2 \%$ & 1 & $9,1 \%$ & 1 & $9,1 \%$ & 11 & $100,0 \%$ \\
\hline Nive muy bajo & 1 & $50,0 \%$ & 0 & $0,0 \%$ & 0 & $0,0 \%$ & 1 & $50,0 \%$ & 0 & $0,0 \%$ & 2 & $100,0 \%$ \\
\hline Total & 12 & $11,9 \%$ & 21 & $20,8 \%$ & 25 & $24,8 \%$ & 25 & $24,8 \%$ & 18 & $17,8 \%$ & 101 & $100,0 \%$ \\
\hline
\end{tabular}

Chi-cuadrado de Pearson $=26,797$ g.l. $=16 \quad p=, 044 *<.05$

Correlación de Spearman $=, 644 * * \quad p=, 000<.05$

*. El estadístico de chi-cuadrado es significativo en el nivel, 05 .

**. La correlación es significativa en el nivel 0,01 (2 colas). 


\section{Tabla 7}

Relación entre las horas de lectura diaria o semanal de los libros del espíritu de profecía con el compromiso cristiano

\begin{tabular}{|c|c|c|c|c|c|c|c|c|c|c|c|c|}
\hline \multirow{3}{*}{$\begin{array}{l}\text { Horas de lectura diaria o } \\
\text { semanal }\end{array}$} & \multicolumn{12}{|c|}{ Compromiso cristiano } \\
\hline & \multicolumn{2}{|c|}{ Nive muy bajo } & \multicolumn{2}{|c|}{ Nivel bajo } & \multicolumn{2}{|c|}{$\begin{array}{c}\text { Nivel } \\
\text { moderado }\end{array}$} & \multicolumn{2}{|c|}{ Nivel alto } & \multicolumn{2}{|c|}{$\begin{array}{l}\text { Nivel muy } \\
\text { alto }\end{array}$} & \multicolumn{2}{|c|}{ Total } \\
\hline & $\mathrm{n}$ & $\%$ & $\mathrm{~N}$ & $\%$ & $\mathrm{n}$ & $\%$ & $\mathrm{n}$ & $\%$ & $\mathrm{n}$ & $\%$ & $\mathrm{n}$ & $\%$ \\
\hline Nivel muy alto & 6 & $8,1 \%$ & 12 & $16,2 \%$ & 19 & $25,7 \%$ & 18 & $24,3 \%$ & 19 & $25,7 \%$ & 74 & $100,0 \%$ \\
\hline Nivel alto & 0 & $0,0 \%$ & 8 & $42,1 \%$ & 4 & $21,1 \%$ & 5 & $26,3 \%$ & 2 & $10,5 \%$ & 19 & $100,0 \%$ \\
\hline Nivel moderado & 2 & $28,6 \%$ & 1 & $14,3 \%$ & 2 & $28,6 \%$ & 2 & $28,6 \%$ & 0 & $0,0 \%$ & 7 & $100,0 \%$ \\
\hline Nivel bajo & 0 & $0,0 \%$ & 0 & $0,0 \%$ & 0 & $0,0 \%$ & 0 & $0,0 \%$ & 0 & $0,0 \%$ & 0 & $0,0 \%$ \\
\hline Nive muy bajo & 3 & $60,0 \%$ & 0 & $0,0 \%$ & 0 & $0,0 \%$ & 2 & $40,0 \%$ & 0 & $0,0 \%$ & 5 & $100,0 \%$ \\
\hline Total & 11 & $10,5 \%$ & 21 & $20,0 \%$ & 25 & $23,8 \%$ & 27 & $25,7 \%$ & 21 & $20,0 \%$ & 105 & $100,0 \%$ \\
\hline
\end{tabular}

Chi-cuadrado de Pearson $=28,914$ g.l. $=16 \quad p=, 004 *<.05$

Correlación de Spearman $=, 521 * *$ y $p=, 000<.05$

*. El estadístico de chi-cuadrado es significativo en el nivel, 05 .

**. La correlación es significativa en el nivel 0,01 ( 2 colas $)$.

\section{Tabla 8}

Relación entre la lectura a nivel literal de los libros del espíritu de profecía con el compromiso cristiano

\begin{tabular}{|c|c|c|c|c|c|c|c|c|c|c|c|c|}
\hline \multirow[b]{3}{*}{ Nivel literal } & \multicolumn{12}{|c|}{ Compromiso cristiano } \\
\hline & \multicolumn{2}{|c|}{ Nive muy bajo } & \multicolumn{2}{|c|}{ Nivel bajo } & \multicolumn{2}{|c|}{$\begin{array}{c}\text { Nivel } \\
\text { moderado }\end{array}$} & \multicolumn{2}{|c|}{ Nivel alto } & \multicolumn{2}{|c|}{$\begin{array}{l}\text { Nivel muy } \\
\text { alto }\end{array}$} & \multicolumn{2}{|c|}{ Total } \\
\hline & $\mathrm{n}$ & $\%$ & $\mathrm{~N}$ & $\%$ & $\mathrm{n}$ & $\%$ & $\mathrm{~N}$ & $\%$ & $\mathrm{n}$ & $\%$ & $\mathrm{~N}$ & $\%$ \\
\hline Nivel muy alto & 0 & $0,0 \%$ & 0 & $0,0 \%$ & 0 & $0,0 \%$ & 0 & $0,0 \%$ & 3 & $100,0 \%$ & 3 & $100,0 \%$ \\
\hline Nivel alto & 0 & $0,0 \%$ & 0 & $0,0 \%$ & 0 & $0,0 \%$ & 5 & $71,4 \%$ & 2 & $28,6 \%$ & 7 & $100,0 \%$ \\
\hline Nivel moderado & 5 & $7,9 \%$ & 9 & $14,3 \%$ & 22 & $34,9 \%$ & 15 & $23,8 \%$ & 12 & $19,0 \%$ & 63 & $100,0 \%$ \\
\hline Nivel bajo & 4 & $15,4 \%$ & 11 & $42,3 \%$ & 3 & $11,5 \%$ & 5 & $19,2 \%$ & 3 & $11,5 \%$ & 26 & $100,0 \%$ \\
\hline Nive muy bajo & 4 & $44,4 \%$ & 1 & $11,1 \%$ & 1 & $11,1 \%$ & 2 & $22,2 \%$ & 1 & $11,1 \%$ & 9 & $100,0 \%$ \\
\hline Total & 13 & $12,0 \%$ & 21 & $19,4 \%$ & 26 & $24,1 \%$ & 27 & $25,0 \%$ & 21 & $19,4 \%$ & 108 & $100,0 \%$ \\
\hline
\end{tabular}

Chi-cuadrado de Pearson $=45,344$ g.l. $=20 \quad p=, 001 * 05$

Correlación de Spearman $=.383 * *$ y $p=, 000<.05$

*. El estadístico de chi-cuadrado es significativo en el nivel, 05 .

**. La correlación es significativa en el nivel 0,01 (2 colas).

\section{Tabla 9}

Relación entre la lectura a nivel inferencial de los libros del espíritu de profecía con el compromiso cristiano

\begin{tabular}{|c|c|c|c|c|c|c|c|c|c|c|c|c|}
\hline \multirow[b]{3}{*}{ Nivel inferencial } & \multicolumn{12}{|c|}{ Compromiso cristiano } \\
\hline & \multicolumn{2}{|c|}{ Nive muy bajo } & \multicolumn{2}{|c|}{ Nivel bajo } & \multicolumn{2}{|c|}{$\begin{array}{c}\text { Nivel } \\
\text { moderado }\end{array}$} & \multicolumn{2}{|c|}{ Nivel alto } & \multicolumn{2}{|c|}{$\begin{array}{c}\text { Nivel muy } \\
\text { alto }\end{array}$} & \multicolumn{2}{|c|}{ Total } \\
\hline & $\mathrm{n}$ & $\%$ & $\mathrm{~N}$ & $\%$ & $\mathrm{n}$ & $\%$ & $\mathrm{~N}$ & $\%$ & $\mathrm{~N}$ & $\%$ & $\mathrm{~N}$ & $\%$ \\
\hline Nivel muy alto & 2 & $8,7 \%$ & 2 & $8,7 \%$ & 5 & $21,7 \%$ & 5 & $21,7 \%$ & 9 & $39,1 \%$ & 23 & $100,0 \%$ \\
\hline Nivel alto & 5 & $13,2 \%$ & 10 & $26,3 \%$ & 6 & $15,8 \%$ & 14 & $36,8 \%$ & 3 & $7,9 \%$ & 38 & $100,0 \%$ \\
\hline Nivel moderado & 2 & $6,9 \%$ & 5 & $17,2 \%$ & 13 & $44,8 \%$ & 2 & $6,9 \%$ & 7 & $24,1 \%$ & 29 & $100,0 \%$ \\
\hline Nivel bajo & 1 & $14,3 \%$ & 3 & $42,9 \%$ & 1 & $14,3 \%$ & 1 & $14,3 \%$ & 1 & $14,3 \%$ & 7 & $100,0 \%$ \\
\hline Nive muy bajo & 3 & $30,0 \%$ & 1 & $10,0 \%$ & 1 & $10,0 \%$ & 4 & $40,0 \%$ & 1 & $10,0 \%$ & 10 & $100,0 \%$ \\
\hline Total & 13 & $12,1 \%$ & 21 & $19,6 \%$ & 26 & $24,3 \%$ & 26 & $24,3 \%$ & 21 & $19,6 \%$ & 107 & $100,0 \%$ \\
\hline
\end{tabular}

Chi-cuadrado de Pearson $=31,105$ g.l. $=16 \quad p=, 013 *<.05$

Correlación de Spearman $=.403 * *$ y $p=, 000<.05$

*. El estadístico de chi-cuadrado es significativo en el nivel ,05.

**. La correlación es significativa en el nivel 0,01 (2 colas). 


\section{Discusión}

En congruencia, Dudley y Commings, en un estudio similar, corrobora que los que leen de manera regular los libros del Espíritu de Profecía (los escritos de Elena G. de White) tienden a tener un rango más alto en su compromiso con Dios, que aquellos que rara vez leen. El estudio detalló que el $85 \%$ de lectores sintieron que tienen una mejor relación íntima con Cristo y el $82 \%$ tenían la seguridad de estar bien con Dios (Des Cumings, 1982)

El presente estudio presenta que cuando un casi $30 \%$ de feligreses mantienen altos sus niveles de lecturas de los libres de Elena de White su compromiso cristiano también se mantiene altos; en contraste también con otros $27 \%$ de feligreses que sus niveles de lecturas de los libros del espíritu de profecía son moderados a niveles bajos igualme nte sus compromisos con el cristianismo se presentan en niveles bajos y moderados respectivamente comprobándose estadísticamente que esta asociación es significativa.

Timm comenta el resultado de una investigación de Bailey Gillespie relacionado con los jóvenes de las escuelas secundarias adventistas del séptimo día en América del Norte. Este estudio reveló que hay una disminución significativa de los que leían a Elena G. White una vez a la semana. La primera encuesta en 1990 indicó que el 13\% de los estudiantes lo hacían, y la segunda en el 2000, diez años después, se encontró que solo el $6 \%$ lo hizo y la tercera en el 2010, incluso un inferior de 2\% (Timm, 2004). Sin embargo, es importante resaltar las formas de lectura que los feligreses mantienen: el estudio manifiesta que un poco más de la mitad $(51.9 \%)$ refiere tener una lectura mecánica en forma regular y sólo un $20 \%$ casi siempre busca conocer el significado de las palabras un aspecto que es resaltante frente a la relación con el compromiso cristiano. Como se observa en la asociación de las tablas de contingencia: mientras los feligreses el $48 \%$ de encuestados mantienen poca lectura de los libros de Elena de White en forma mecánica sus niveles de compromiso cristiano se mantienen altos un aspecto que la correlación de Spearman contrasta evidenciando una relación directa muy significativa más allá de la media; es decir que un grupo de los encuestados evidencia que mantienen niveles de lectura mecánica y sin embargo mantienen compromiso cristiano

De la misma forma, otra forma también que puede estar relacionado con este aspecto espiritual es la lectura rápida donde el $26.9 \%$ acredita hacerlo regularmente y un 26,9\% casi siempre lo hace rápido su lectura del espíritu de profecía leyendo casi siempre sólo lo que le interesa. Al contrastar la laxabilidad en la forma de leer los libros de Elena de White con la frecuencia que se da en sus lecturas se podría inferir una baja profundidad 
en su comprensión considerando que el 50\% lee los libros de la hermana White constantemente o al menos una vez o más días a la semana eligiendo un libro al año para su lectura y lo hace al menos una hora por día y cabe resaltar que un 36\% también lo hace pero esporádicamente y que probablemente usan algunas horas en la semana para su lectura.

Las tablas de contingencia de asociación se contrasta los datos evidenciando que mientras que el $40 \%$ de feligreses de encuestados su frecuencia de lectura de los libros de Elena de White es baja, sus niveles de compromiso cristiano se mantienen altos; un aspecto que la correlación de Spearman contrasta evidenciando una relación directa muy significativa en forma considerable ; es decir que un grupo de los encuestados evidencia que mantienen una frecuencia baja en la lectura y sin embargo mantienen compromiso cristiano altos. A diferencia de un grupo encuestados $30 \%$ que mantienen horas de lectura diaria o semanal evidencia niveles altos de compromiso cristiano un aspecto corroborado con el coeficiente de correlación de Spearman en forma directa y significativa; es decir mientras más horas de dedicación a la lectura de los libros del Espíritu de Profecía le estén dando su compromiso cristiano mantienen altos.

Dichos resultados evidencian de forma descriptiva una realidad actual de la feligresía adventista: mientras la forma de lectura es superficial y rápida y la frecuencia es escasa; las tablas de contingencia muestran dos grupos que mientras algunos mantienen ese polo negativo y laxativo en la lectura del espíritu de profecía su compromiso cristiano se mantiene alto igual que los que mantienen el polo positivo de interiorización de la lectura de los libros de Elena de White también presentan niveles altos. Sin embargo, la correlación resalta un coeficiente directo y significativo en la forma, frecuencia y horas de lectura con el compromiso cristiano que se evidencia en la comunión, relacionamiento y misión.

Por otro lado, dicha realidad dicotomizada en la lectura de los libros de Elena de White: mientras algunos lo hacen diligentemente comprendiendo y con alta frecuencia mientras otros lo realizan en forma "flexible" se refleja en los niveles de comprensión de sus lecturas como manifiesta este estudio que un $52.8 \%$ de los encuestados no comprende lo que estaba ocurriendo cuando Elena de White quien en una de sus visiones profetiza y sostiene la Biblia con sus manos. Este aspecto refleja que el 58.3\% de encuestados acreditan entender los escritos del Espíritu de profecía en forma literal a diferencia de un $57.4 \%$ lo hace en forma inferencial o, dicho de otro modo; traspolando su interpretación a un contexto específico o aun significado en particular. 
Las tablas de contingencia complementan dichos resultados descriptivos; mientras que un grupo de encuestados $49 \%$ interpretan la lectura literal de los libros del espíritu de profecía en forma regular y a pesar de ello su compromiso cristiano se mantiene de niveles moderados hacia niveles altos. El coeficiente de Spearman demuestra una relación casi media en forma directa y significativa; es decir a mayor interpretación inferencial mayor compromiso cristiano.

Analizar estos resultados muestra que la población adventista se muestra dividida en un aspecto importante que puede tener un impacto en el compromiso cristiano y desarrollo de la fe cristiana adventista. Como refieren los resultados del estudio, el nivel de compromiso cristiano de los encuestados muestra que un $31 \%$ mantienen niveles bajos en su compromiso cristiano y un $24,1 \%$ un compromiso cristiano mediocre afectando su comunión, relacionamiento y misión. Dicho aspecto es vital ser considerando en vista que las escrituras bíblicas reflejan que el compromiso cristiano de sus miembros está basado en el conocimiento de Dios como el único Dios verdadero (Juan 17:3). Ese "conocimiento de Dios y de su voluntad expresada en Cristo es vital para el crecimiento personal y del cuerpo-iglesia aquí en esta tierra" (Scholtus, 2005). Además, el apóstol Pablo, para desarrollar la fe, es necesario un mayor compromiso con la palabra de Dios. Él lo afirma en los siguientes términos, la "fe es por el oír, y el oír, por la palabra de Dios" (Romanos 10:17). Finalmente refiere Barnes Joselyn, considerando el aporte de los escritos de Elena G. de White sobre la educación; en su tesis Doctoral menciona que, "los consejos de Elena G. de White no pueden tomarse a la ligera hoy por ningún miembro de la IASD, los principios educacionales de White han sido y son la base para el desarrollo..." (Barnes, 1982)

En conclusión se determina que existe relación significativa y positiva entre la lectura de los escritos de Elena G. de White y compromiso cristiano de los feligreses adventistas en Chone. También, se puede afirmar que existe relación negativa entre la lectura rápida con el compromiso cristiano de los feligreses adventistas de Chone, existe correlación media e inversa entre las variables.

\section{Referencias}

Barnes, C. J. (1982). 'Physical Work as an Integral part of Education at Oakwood College in Light of Ellen G. White's Writings" (Thesis Doctoral). Wayne State University. 
Burchfield, L. S. (1996). "Adventist Religious Experience, 1816-1868: A Comparison of William Miller and Ellen White". (Thesis Doctoral). Southern Baptist Theological Seminary.

Burt, M. D. (2013). Elena G. de White y el surgimiento del adventismo sabatista. En Quispe, G., Burt, M. D. y Timm, A. (eds). Legado Adventista: Un panorama histórica y teológico del adventismo. Lima: Ediciones Universidad Peruana Unión.

Chávez, D. A. (2012). El culto de los Niños. Estrategias para el cumplimiento de la misión, 9, 2012.

Coon R., (1983). A gift of light. Washington, DC: Review and Herald Publishing Association.

Cummings Jr., D. \& Dudley, R. L. (1982). Who reads Ellen White: Do church members who regularly read the writings of Ellen White differ significantly from those who seldom do? Ministry Magazine, October.

Douglass, H. E. (2003). Mensajera del Señor: Ministerio profético de Elena G. de White, Buenos aires: Asociación Casa Editora Sudamericana.

Gilleespie, V. \& Bailey E. T. (2004). Valuegenesis, Ten Years Later: A Study of Two Generations, California: Hancock Center.

Iriarte, A., https://www.milenio.com/opinion/carlos-iriarte/red-social/la-era-de-lainmediatez (Consultado el 13 de junio, 2019)

Kidder, J. S. (2009). La fuerza de la amistad. El ministerio Adventista, marzo- abril.

Knight, G. R. (2004). Como leer Elena de White, Florida, EEUU: Asociación Publicadora interamericana.

Richard, D. (2013). Investigar: una actividad sencilla, enriquecedora y factible, Lima: Editorial Imprenta Unión.

Presser N. (2011). El poder de la palabra de Dios. Revista adventista, febrero.

Sampieri, R. H. (2010). Metodología de la investigación. Lima: Empresa Editora El Comercio S.A.

Timm, A. R. (2004). Asuntos contemporáneos en la teología Adventista (Ponencia, asignatura). Lima, Perú.

Verdu, Lara Llopis, La cultura de la inmediatez. https://elpais.com/elpais/2019/03/30/opinion/1553960918_319282.html,(Consul tado el 13 de junio, 2019)

White, E. G., Step to Christ. California, USA: Pacific Press.

Zylstra, S. H. (2016). What the Latest Bible Research Reveals About Millennials. Christianity Today, $16 \quad$ de https://www.christianitytoday.com/news/2016/may/what-latest-bibleresearchreveals-about-millennials.html (consultado: 19 de julio, 2018) 